\title{
Introduction aux réseaux de symptômes en psychopathologie
}

$>$ Nous présentons dans cet article les grands principes méthodologiques des réseaux de symptômes en psychopathologie. Ceux-ci pourraient constituer une approche alternative au manuel diagnostique et statistique des troubles mentaux (DSM) et aux Research Domain Criteria (RDoC), sans les exclure pour autant. Les réseaux de symptômes dépassent ou contournent certaines limites de ces classifications, mais leur avenir est encore incertain : ils devront relever des défis épistémologiques et méthodologiques, tout en parvenant à convaincre la communauté de chercheurs et de cliniciens en santé mentale de leur utilité et de leur qualité. <

La publication du DSM-III en 1980 (Diagnostic and Statistical Manual ; American Psychiatric Association) [1] fournit à la psychiatrie une nosologie radicalement différente de toutes les autres classifications antérieures des troubles mentaux. Ses catégories diagnostiques furent construites par la réunion des critères de Feighner et al., décrits dans les suites des travaux de l'źcole de Saint Louis [2], et de définitions opérationnelles validées par les chercheurs de l'American Psychiatric Association (les Research Diagnostic Criteria, ou RDC, [3]). Les méthodes d'intervention structurées et les nouvelles technologies allaient soutenir le développement de cette classification émergente. La validation méthodologique et catégorielle des troubles mentaux devenait un horizon accessible.

Actuellement dans sa cinquième version, le DSM cible des manifestations cliniques (les signes et symptômes que l'on rassemble sous le terme de «sémiologie ») regroupées sous la forme d'entités nosologiques ${ }^{1}$. Leur validité et leur fiabilité sont respectivement statistiquement insuffisantes et cliniquement discutables [4]. Rappelons succinctement que la validité d'une classification permet d'affirmer que la classification mesure bien ce qu'elle est censée mesurer, et garantit que les catégories reflètent réellement une maladie mentale

Des sous-ensembles de maladies au sein d'une classification.

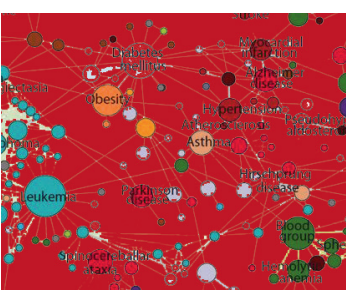

Service de neurologie et psychiatrie, CHU Grenoble-Alpes, domaine de La Merci, 38000 La Tronche, France. Adresse actuelle : Service de Psychiatrie du Centre Hospitalier Universitaire de Saint-Étienne, Hôpital de Bellevue, 25 boulevard Pasteur, 42000 Saint-Étienne, France. chrisgauld@hotmail.fr

existante. La fiabilité d'une classification permet un accord entre les examinateurs et dans le temps [5], entraînant un meilleur partage de l'information, et donc la génération de progrès scientifiques [6]. Audelà de ces imperfections méthodologiques, c'est la logique même du DSM qui pourrait être critiquable : ce sont des catégories cliniques qui dicteraient le choix d'un marqueur psychophysiologique sur lequel s'appuierait une prise en charge clinique (par exemple, médicamenteuse). Dans un article de 2017, Zachar et Kendler proposaient une extension de la description des troubles psychiatriques non seulement vers la prise en compte des caractéristiques phénotypiques non incluses dans les critères diagnostiques du DSM-5, mais également vers une restructuration de ces critères, afin qu'ils soient plus en cohérence avec «la recherche sur les circuits cérébraux et d'autres facteurs agissant à plusieurs niveaux d'analyse » [7]. La nécessité de stabiliser les classifications de la psychiatrie sur d'autres fondations que les seules données sémiologiques s'est vue concrétisée en 2009 lors de la constitution d'un groupe de travail en recherche translationnelle. Celui-ci, à l'initiative de la constitution des critères pour des domaines de recherche (Research Domain Criteria, RDoC), a proposé de considérer les niveaux macroscopiques et microscopiques au sein d'une même matrice. Cette matrice constitue un cadre d'analyse reflétant les altérations de multiples systèmes cérébraux qui interviennent dans des processus mentaux particuliers (en lignes) en fonction des différents niveaux d'analyses du vivant (en colonnes) [8]. Les praticiens de santé mentale et les chercheurs qui s'intéressent à la psychiatrie ont la possibilité de manier plusieurs classifications. Leur évolution en parallèle doit avoir un intérêt pratique, afin d'échapper à la simple «botanisation des troubles mentaux », pour reprendre l'expression d'Haslam [9]. Pour en comprendre ces intérêts cliniques et épistémiques, nous replacerons, dans un premier temps, les RDoC dans leur contexte actuel. Nous présenterons ensuite l'approche des réseaux des symptômes en psychopathologie, qui dépasse les limites des RDoC sans rejeter pour autant les classifications sémiologiques, comme le DSM-5. 


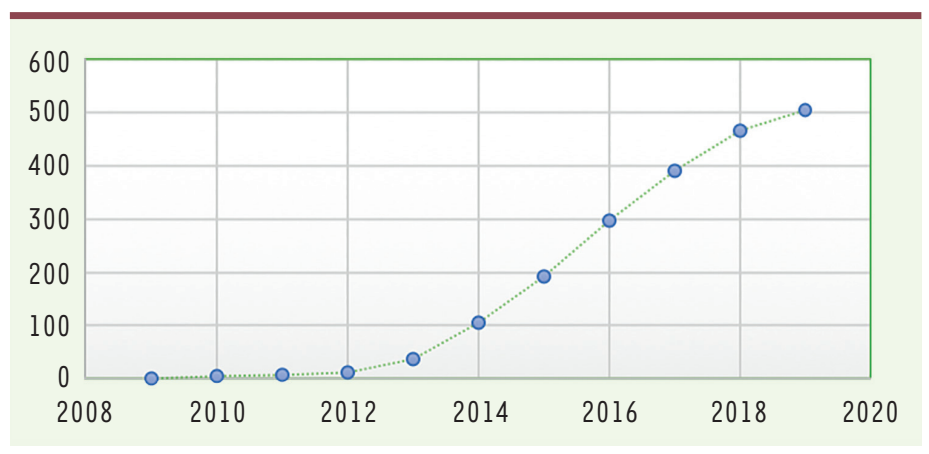

Figure 1. Nombre de publications «RDoC-compatibles 》 référencées sur Pubmed/Medline, recherchées avec le terme «RDoC » en fonction des années. À noter que ce calcul n'est pas exhaustif, mais permet de voir l'augmentation relative des références au terme $\mathrm{RDoC}$ au fil des années.

\section{Les Research Domain Criteria}

Selon la définition du National Institute for Mental Health (NIMH) aux États-Unis, le projet RDoC entend réconcilier les domaines neuroscientifiques du fonctionnement du cerveau avec les différentes échelles d'analyse, qui vont des gènes aux mesures auto-rapportées (par exemple, la communication sociale ou l'attachement) en passant par les circuits neuronaux (par exemple, les circuits dopaminergiques) [10]. L'idée sous-jacente de ce projet est d'utiliser des bases neurobiologiques et comportementales pour proposer de nouveaux traitements adaptés aux dysfonctions mentales [11]. Les RDoC ont donc des implications ontologiques (elles refondent de manière dimensionnelle la compréhension des troubles mentaux sur des dysfonctionnements cérébraux), méthodologiques (des systèmes cérébraux peuvent être utilisés pour étudier le fonctionnement psychopathologique) et cliniques (les RDoC pourraient à terme aboutir à une classification) [12]. Les RDoC encouragent donc certaines priorités dans le soin psychiatrique : un mode de collecte et d'analyse de données particulier, des préférences entre divers instruments de mesure et entre niveaux d'explication [13]. Mais en même temps, ils découragent d'autres initiatives, le NIMH engageant plus ou moins explicitement les psychiatres à se concentrer sur les données du contexte neuroscientifique. Comme l'écrivaient Demazeux et Pidoux en 2015, si le projet RDoC a d'abord été présenté comme "un projet de recherche complémentaire au système DSM », il est vite apparu comme « un projet en réalité alternatif, dont le développement est conditionné par sa capacité à stimuler la recherche psychiatrique dans la perspective originale qu'il entend ouvrir » $[12](\rightarrow)$ $(\rightarrow)$ Voir le Forum de S. Demazeux et V. Pidoux, $m / s n^{\circ} 8-9$, août-septembre 2015, page 792
L'initiative des $\mathrm{RDoC}$ restant relativement récente, il est vrai que ses succès et ses échecs ne pourront être jugés qu'une fois qu'ils auront été bien compris [14]. La Figure 1 montre certes une augmentation des références au RDoC, mais dix ans exactement après le début de son élaboration, il est encore délicat d'affirmer si les ambitions de ce programme sont aussi fécondes qu'elles ne veulent le laisser penser. Étant donné la nature encore rudimentaire des données reliant les mesures de la fonction cérébrale à la psychopathologie, la production d'outils cliniquement utiles pourrait prendre beaucoup de temps [15]. Mais la collaboration entre philosophes et psychiatres ne pourra devenir que plus fructueuse face à l'incertitude des modèles de classification qui émergent dans le paysage de la santé mentale.

En définitive, les critiques des $\mathrm{RDoC}$ portent sur quatre arguments principaux [16]: un argument scientifique, selon lequel les fondateurs des $\mathrm{RDoC}$ postulaient que la science possédait suffisamment de connaissances sur le fonctionnement cérébral pour qu'un projet de fondation de la psychiatrie sur des marqueurs biologiques puisse être concevable [17]; un argument clinique, selon lequel l'utilisation du RDoC dans le quotidien des psychiatres n'est pas encore d'actualité, pour des raisons aussi bien conceptuelles que sémantiques [18] ; un argument structurel, qui porte sur son apparent réductionnisme [19]; et un argument épistémologique, attestant du fait que le phénotype psychiatrique n'est jamais le résultat d'un unique marqueur.

Il s'avère que cette dernière critique (épistémologique) mériterait d'être développée. En effet, la matrice proposée par les RDoC explore de manière dimensionnelle différents niveaux d'analyse, mais ces niveaux ne sont pas causalement reliés entre eux. La possibilité de caractériser un lien de causalité entre ces niveaux est pourtant envisageable. Autrement dit, lorsqu'un des éléments de la matrice agit sur un autre, cette action pourrait être modélisée dans un réseau. Pour cela, il a fallu historiquement conjuguer les apports des théories des réseaux [20] et de la biologie des systèmes [21]. Nous allons ainsi explorer la manière avec laquelle cette dénommée «psychiatrie des systèmes (ou des réseaux) 》 pourrait renforcer les apports épistémologiques, cliniques et thérapeutiques des RDoC, sans rejeter pour autant les classifications fondées sur la sémiologie [22].

\section{La psychiatrie des systèmes}

«Rien ne s'obtient sans computation, même la plus extraordinaire des intuitions. »²

La médecine individualisée distingue une médecine «stratifiée » d'une médecine des systèmes [23]: la première cherche à déterminer des marqueurs personnalisés pour des sous-groupes d'individus (comme le fait le RDoC); la seconde développe des outils pour relier ces marqueurs au sein d'un réseau.

La médecine des systèmes est donc une médecine stratifiée qui, en étudiant les relations entre des com-

${ }^{2}$ Morin $\varepsilon$. La Méthode, tome III, La Connaissance de la connaissance. Paris : Seuil 1986 : p. 147. 


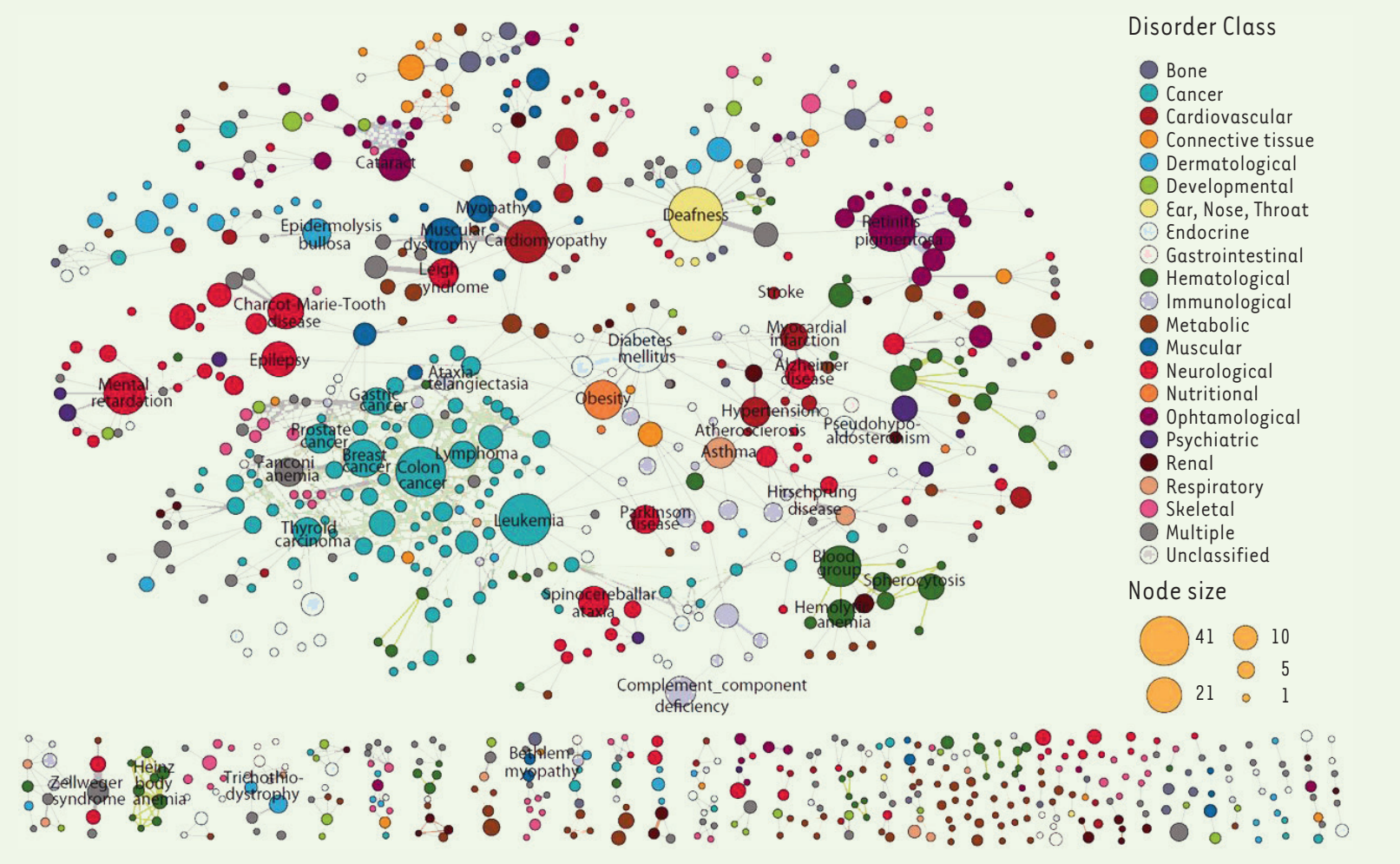

Figure 2. Diseasome : le réseau topologique des maladies humaines. La taille des nœuds est proportionnelle au degré de connectivité. Le code couleur permet de distinguer différentes classes de maladie (d'après [26]).

posants d'un système organique, propose d'analyser finement les différences entre individus et entre catégories [24]. Cette médecine des réseaux détermine des principes généraux sur l'organisation et les propriétés des individus : comme le montre la Figure 2, elle est capable de tracer les interactions entre les pathologies [25].

Appliquée à la psychiatrie, l'approche en réseaux dépasse la vision de la sémiologie épidémiologique (qui s'intéresse uniquement aux signes pertinents sur des échantillons statistiques, comme le DSM) et à celle de la physio-biologie des connexions, des molécules ou de la génétique (qui s'intéresse uniquement à l'analyse isolée d'un ensemble de composants du système, comme le projet RDoC). Son objectif est d'étudier les interactions entre les composants des systèmes humains. La détermination de tels réseaux relève d'un travail qui est en cours de réalisation en psychiatrie. On peut citer à ce propos le projet PsychoSystems ${ }^{3}$, qui travaille sur les réseaux de symptômes de la psychopathologie. Ce modèle classificatoire, à la fois destiné à la clinique [27] et à la recherche [28], s'appuie sur une intégration de la sémiologie et des autres niveaux d'analyse dans un réseau unique pour un individu ou un trouble psychiatrique.

Ce modèle postule l'existence d'un réseau causal, c'est-à-dire un réseau dans lequel une entité pourrait expliquer la survenue ou le maintien d'une autre entité appartenant également au réseau. Ce réseau causal inclus à la fois des éléments sémiologiques (par

${ }^{3}$ http://psychosystems.org/ exemple, des reviviscences) des éléments environnementaux (par exemple, un facteur de stress aigu) et des éléments biologiques (par exemple, des gènes). C'est l'ensemble des nœuds et des interactions formant le réseau qui rend compte du trouble mental observé (par exemple, un état de stress post-traumatique). Les troubles mentaux peuvent donc être compris comme des états stables de réseaux de symptômes fortement connectés [29]. Comme nous allons le développer, ces notions permettent d'étudier plus aisément des facteurs d'émergence, de résilience, de vulnérabilité, de comorbidité et de responsabilité causale [27]. Comme l'illustre la Figure 3 par l'exemple de la dépression, il est possible de représenter les modèles du DSM, des RDoC et des réseaux de symptômes en en présentant leur représentation de ce qu'est une «explication» d'un trouble mental. Ainsi, à l'image du DSM, la catégorie clinique est l'effet commun d'un ensemble de symptômes («mécanisme à effet commun »). À l'instar des $\mathrm{RDoC}$, une cause commune explique un ensemble de symptômes cliniques («mécanisme à cause commune »). Enfin, comme cela est retrouvé dans les réseaux de symptômes, le mécanisme d'influence causale entre les symptômes est réciproque (« mécanisme à effet réciproque »). 
Spécificités épistémologiques

Dépasser la question de la variable latente

Redistribuer la causalité

Modéliser la complexité

Éviter le réductionnisme

\section{Conséquences cliniques}

Mettre en relation mutualiste la sémiologie et les paramètres physio-biologiques

Intégrer différentes échelles d'exploration

Fournir une cartographie («simulation ») personnalisée

Laisser une grande part à la sémiologie clinique

Tableau I. Caractéristiques épistémologiques et implications cliniques des réseaux de symptômes en psychopathologie.

Les modèles en réseau de symptômes évitent certaines problématiques des classifications sémiologiques actuelles et des RDoC.

D'abord, ils contournent la problématique de la variable latente: les troubles mentaux sont constitués par des interactions directes entre symptômes [31]. En pratique, ces variables sous-jacentes ont été peu identifiées en psychiatrie, notamment parce que les différentes versions des DSM, du fait de leur «athéorisme », cherchaient à se dégager de toute implication biologique.

De ce fait, ce type de réseaux permet de redistribuer la part de causalité (c'est-à-dire l'implication) d'un symptôme donné ou d'une anomalie infra-clinique dans le phénotype. Autrement dit, la causalité est répartie au sein du réseau, rejoignant les thèses du pluralisme intégratif de Kendler [32].

Ils sont donc particulièrement intéressants lorsqu'il s'agit de discuter l'intrication de facteurs causaux [33]. Comme nous l'avons dit, dans le DSM, ces facteurs causaux ne sont pas inclus dans les critères diagnostiques. En cohérence avec les hypothèses de Dennett, les réseaux de symptômes suggèrent que les troubles mentaux présentent des facteurs biologiques et psychologiques étroitement imbriqués dans une «boucle à retour d'information »: ni le niveau psychologique ni le niveau biologique ne peut prétendre à une priorité causale ou explicative. Ils incorporent la complexité inhérente à la psychiatrie [34].

Enfin, en lien avec ce pluralisme, ils évitent le réductionnisme naïf dans la recherche en psychopathologie [35]. Par exemple, la force des relations dans le réseau dépendra de la pondération des contextes culturels et historiques aussi bien que de celle des mécanismes biologiques. Le Tableau / résume les quatre caractéristiques épistémologiques des réseaux de symptômes en psychopathologie que nous venons de décrire.

$\varepsilon$ n pratique clinique, les réseaux de symptômes n'excluent pas les modèles classificatoires existants, que ce soit le DSM (duquel il récupère les catégories) ou les $\mathrm{RDoC}$ (dont il utilise les niveaux d'analyse). Dans une classification polythétique telle que celle du DSM, ils permettent d'aider à définir quels sont les critères les plus valides pour définir un trouble. Ils servent également de soutien à ces cadres théoriques en tant qu'outils complémentaires [36]. Par exemple, ils permettent de comparer les réseaux de personnes porteuses de troubles du spectre de l'autisme lorsque ce trouble est associé ou non à une déficience intellectuelle [37], de comparer un même ensemble de symptômes chez deux individus, d'étudier à intervalles réguliers l'efficacité d'un traitement [28], d'aider à distinguer les comorbidités ou les facteurs de risque [38], etc.

Mais en tant que classification, les réseaux de symptômes en psychopathologie sont loin d'être inscrits dans la pratique courante. Ainsi, au premier abord, cette approche pouvait apparaître comme une alternative naissante aux classifications contemporaines. Mais de nombreux obstacles sont encore à surmonter. D'abord, en pratique, l'utilisation des réseaux de symptômes en clinique semble être aussi délicate à déployer (par exemple, du fait des contraintes liées à la modélisation informatique) que difficile à justifier (par exemple, concernant la plus-value par rapport aux classifications employées). Ensuite, en théorie, les débats sur le sujet dans la littérature spécialisée sont encore animés, allant de la défense pour leur replicabilité [39] à celle portant contre son réductionnisme [37], défenses qui pourraient conduire à la récupération de notions issues de la médecine personnalisée et d'autres disciplines, comme celles de driver adaptatif et d'addiction étiogénique [40]. Dans le fond, il est en effet bien légitime de se demander si les réseaux de symptômes pourront surmonter des problématiques inhérentes à leur propre constitution : comment vontils parvenir à privilégier un ensemble de mécanismes par rapport à un autre? Comment vont-ils délimiter les catégories diagnostiques ("carving the nature at its joint», selon l'expression célèbre des taxonomistes)? Comment pourront-ils construire une nosologie formant un tout cohérent?

\section{Conclusion}

Étant donné la présence d'étiologies multifactorielles et complexes dans le domaine psychiatrique, il semble approprié de présenter une méthode de classification qui intègre toutes ces dimensions. II paraît nécessaire de s'appuyer sur les différents cadres de travail théoriques et modèles de soin qui circulent aujourd'hui entre les disciplines qui composent et côtoient la psychiatrie. 

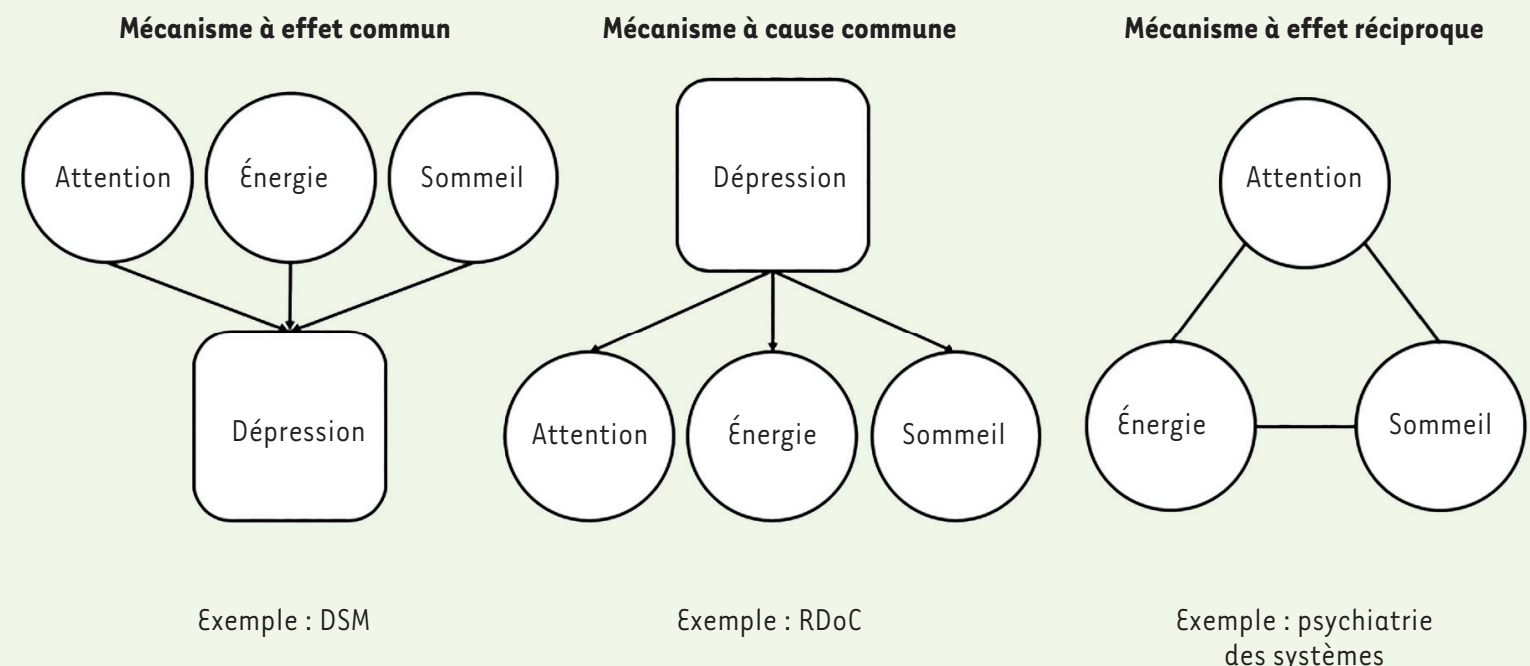

Figure 3. Trois mécanismes de causalité dans trois modèles de psychopathologie dont le paradigme explicatif est divergent. À gauche, le « mécanisme à effet commun » représente la manière avec laquelle trois symptômes déterminent la catégorie clinique de la dépression. Au centre, le «mécanisme à cause commune » indique la manière dont une cause peut agir en tant que mécanisme explicatif de ces trois mêmes symptômes. Le schéma de droite représente la causalité réciproque des symptômes, sans « cause » ni « effet » communs (nommés « variables latentes »), tel que cela est retrouvé dans les réseaux de symptômes (schéma issu de [30]).

Une étude de 2012 portant sur les préférences de 869 internes français de psychiatrie rapportait que, de manière unanime, l'unique psychothérapie qui n'était pas contestée était la systémie. Pourquoi ? Car à la différence de la psychanalyse ou des thérapies cognitivo-comportementales, elle inclut différents niveaux d'analyses, comme le concluent les auteurs [41]. La psychiatrie des systèmes, certes loin des méthodes de la psychothérapie systémique, possède tout de même des fondations épistémologiques (et ontologiques) communes avec celle-ci. En tout cas, elle pourrait être un des vecteurs de la réconciliation entre des tendances psychiatriques divisées dans le paysage français.

Mais sans doute est-il bien trop tôt pour mesurer les promesses d'un tel projet de modélisation. De là à être acceptés communément et par tous en clinique, les réseaux de symptômes ont encore du chemin à faire. Les psychiatres sont-ils prêts à résumer un individu ou une pathologie à un module connecté ? La recherche est-elle prête à modifier ses méthodes d'investigation et à sortir du cadre du DSM ? On peut cependant espérer que si la coordination entre psychiatrie et philosophie continue de se faire, les praticiens en santé mentale se rendront mieux compte des implications et des conséquences d'un autre modèle du trouble mental. Cependant, il ne faudrait pas qu'une telle conception du soin agisse comme une nouvelle «boîte de Pandore » offerte à la psychiatrie, qui contienne autant d'entraves qu'elle n'apporte d'innovations. $\diamond$

\section{SUMMARY}

Introduction to symptom networks in psychopathology

In this article, we present the main methodological principles of symptom networks in psychopathology. It is a topological approach linking entities from different scales of analysis of an individual (from genetics to behavior, via cerebral connectivity). They are an alternative to the Diagnostic and Statistical Manual of Mental Disorders (DSM) and Research Domain Criteria (RDoC), but they do not exclude them. Symptom networks exceed or circumvent some limits of these classifications. Furthermore, they contribute to the stratification and organization of these nosologies. Behind the originality of its methodology, this program proposes a redefinition of mental illness which modifies the conception of psychiatry. But their future is still uncertain: they must take on an epistemological and methodological challenge. At the same time, they have to convince the community of mental health researchers and clinicians of their utility and value. $\diamond$

\section{LIENS D’INTÉRÊT}

L'auteur déclare n'avoir aucun lien d'intérêt concernant les données publiées dans cet article.

\section{RÉFÉRENCES}

1. American Psychiatric Association. Diagnostic and statistical manual of mental disorders, DSM-5, $5^{\mathrm{e}}$ ed. Arlington, VA : American Psychiatric Publishing, 2013.

2. Feighner JP, Robins $\varepsilon$, Guze SB, et al. Diagnostic criteria for use in psychiatric research. Arch Gen Psychiatry $1972 ; 26: 57-63$.

3. Spitzer RL, Endicott J, Robins $\varepsilon$. Research diagnostic criteria. New York state psychiatric Institute, biometrics research. New York : New York State Department of Mental Hygiene, 1975.

4. Whooley 0. Diagnostic ambivalence: psychiatric workarounds and the Diagnostic and statistical manual of mental disorders. Sociol Health IIIn $2010 ; 32: 452-69$ 


\section{RÉFÉRENCES}

5. Cohen, J. A coefficient of agreement for nominal scales. Educational Psychological Measurement $1960 ; 20: 37-46$.

6. Hempel CG. Scientific explanation. Voice of America 1966.

7. Zachar P, Kendler KS. The philosophy of nosology. Annu Rev Clin Psychol 2017 ; 13 : 49-71.

8. Cuthbert BN, Insel TR. Toward the future of psychiatric diagnosis: the seven pillars of RDoC. BMC Med $2013 ; 11: 126$.

9. Haslam N. Kinds of kinds: a conceptual taxonomy of psychiatric categories. Philosophy Psychiatry Psychology $2002 ; 9$ : 203-17.

10. Cuthbert BN, Insel TR. Toward new approaches to psychotic disorders: the NIMH research domain criteria project. Schizophr Bull $2010 ; 36: 1061-2$

11. Kozak MJ, Cuthbert BN. The NIMH research domain criteria initiative: background, issues, and pragmatics. Psychophysiology $2016 ; 53: 286-97$.

12. Demazeux S, Pidoux V. Le projet RDoC. La classification psychiatrique de demain ? Med Sci (Paris) $2015 ; 31: 792-6$.

13. Sullivan JA. Coordinated pluralism as a means to facilitate integrative taxonomies of cognition. Philosophical Explorations 2017 ; 20 : 129-45.

14. Tabb K, Schaffner KF, Kendler KS, Parnas J. Causal pathways, random walks and tortuous paths: Moving from the descriptive to the etiological in psychiatry. Philosophical Issues Psychiatry IV Psychiatric Nosology ; 2017 ; 342-60.

15. Kirmayer LJ, Crafa D. What kind of science for psychiatry? Front Hum Neurosci $2014 ; 8: 435$

16. Carpenter WT. RDoC and DSM-5: what's the fuss? Schizophr Bull 2013 ; 39 : 945-6.

17. Micoulaud-Franchi JA, Quiles C, Batail JM, et al. Vers une approche physiologique de la sémiologie en psychiatrie. Partie 1 : approches RDC, DSM, RDoC et HiTOP. Ann Med-Psycholo Rev Psychiatr $2019 ; 177: 282-8$.

18. Etkin A, Cuthbert B. Beyond the DSM: development of a transdiagnostic psychiatric neuroscience course. Academic Psychiatry $2014 ; 38$ : 145-50.

19. Faucher L, Goyer, S. RDoC: Thinking outside the DSM box without falling into a reductionist trap. In : The DSM-5 in perspective. Dordrecht : Springer, 2015 : 199-224.

20. Barabási AL, Bonabeau E. Scale-free networks. Scientific American $2003 ; 288: 60-9$

21. Kitano H. Systems biology: a brief overview. Science 2002 ; 295 : 1662-14

22. Dumas $G$, Cermolacce M, Batail JM, et al. Vers une approche physiologique de la sémiologie en psychiatrie. Partie 2 : perspectives offertes par la biologie systémique. Ann Med-Psycholo Rev Psychiatr 2019; $177: 289-94$

23. Guchet X. La médecine personnalisée, un essai philosophique. Paris : Les Belles Lettres, 2016.

24. Borsboom D, Cramer A0. Network analysis: an integrative approach to the structure of psychopathology. Annu Rev Clin Psychologie 2013 ; 9 : 91-121.

25. Woodward J. Mechanistic explanation: its scope and limits. Proc Aristotelian Society (Suppl Volumes) $2013 ; 87: 39-65$

26. Goh Kl, Cusick ME, Valle D, et al. The human disease network. Proc Natl Acad Sci USA 2007 ; 104 : $8685-90$
27. Blanken TF, Van Der Zweerde T, Van Straten A, et al. Introducing network intervention analysis to investigate sequential, symptom-specific treatment effects: a demonstration in co-occurring insomnia and depression. Psychotherapy Psychosomatics $2019 ; 88: 52-4$.

28. Borsboom D. A network theory of mental disorders. World Psychiatry 2017 ; $16: 5-13$.

29. Fried हl, Cramer A0. Moving forward: challenges and directions for psychopathological network theory and methodology. Perspectives Psychological Science 2017 ; 12 : 999-1020.

30. Kruis J, Maris G. Three representations of the Ising model. Sci Rep 2016; 6 : 34175

31. Borsboom D, Mellenbergh GJ, Van Heerden J. The theoretical status of latent variables. Psychological Rev $2003 ; 110: 203$

32. Kendler KS. Toward a philosophical structure for psychiatry. Am J Psychiatry $2005 ; 162: 433-40$

33. Cramer A0, Waldorp LJ, Van Der Maas HL, Borsboom D. Comorbidity: a network perspective. Behav Brain Sci 2010 ; 33 : 137-50.

34. Dennett DC. Brainstorms: philosophical essays on mind and psychology. Cambridge (MA), États-Unis : MIT Press, 2017.

35. Borsboom D, Cramer A0, Kalis A. Brain disorders? Not really... Why network structures block reductionism in psychopathology research. Behav Brain Sci 2018 ; Jan 24 : 1-54.

36. Darrason M. Médecine de précision et médecine des systèmes : la médecine personnalisée se trompe-t-elle de cible ? Lato Sensu - Revue de la Société de Philosophie des Sciences 2017 ; 4 : 66-82.

37. Anderson GM, Montazeri F, de Bildt A. Network approach to autistic traits: group and subgroup analyses of ADOS item scores. J Autism Dev Disord $2015 ; 45: 3115-32$

38. Isvoranu AM, Borsboom D, van Os J, Guloksuz S. A network approach to environmental impact in psychotic disorder: brief theoretical framework. Schizophr Bull $2016 ; 42: 870-3$

39. Borsboom D, Fried El, Epskamp S, et al. False alarm? A comprehensive reanalysis of evidence that psychopathology symptom networks have limited replicability, by Forbes, Wright, Markon, and Krueger. J Abnorm Psychol 2017 ; 126 : 989-99.

40. Tonon $\mathrm{G}$. From oncogene to network addiction: the new frontier of cance genomics and therapeutics. Future Oncol $2008 ; 4$ : 569-77.

41. Van Effenterre A, Azoulay M, Champion F, Briffault X. Initial training in psychotherapy for psychiatrists in France: results of a national survey. Encephal 2013; $39: 155-64$

\section{TIRÉS À PART}

C. Gauld

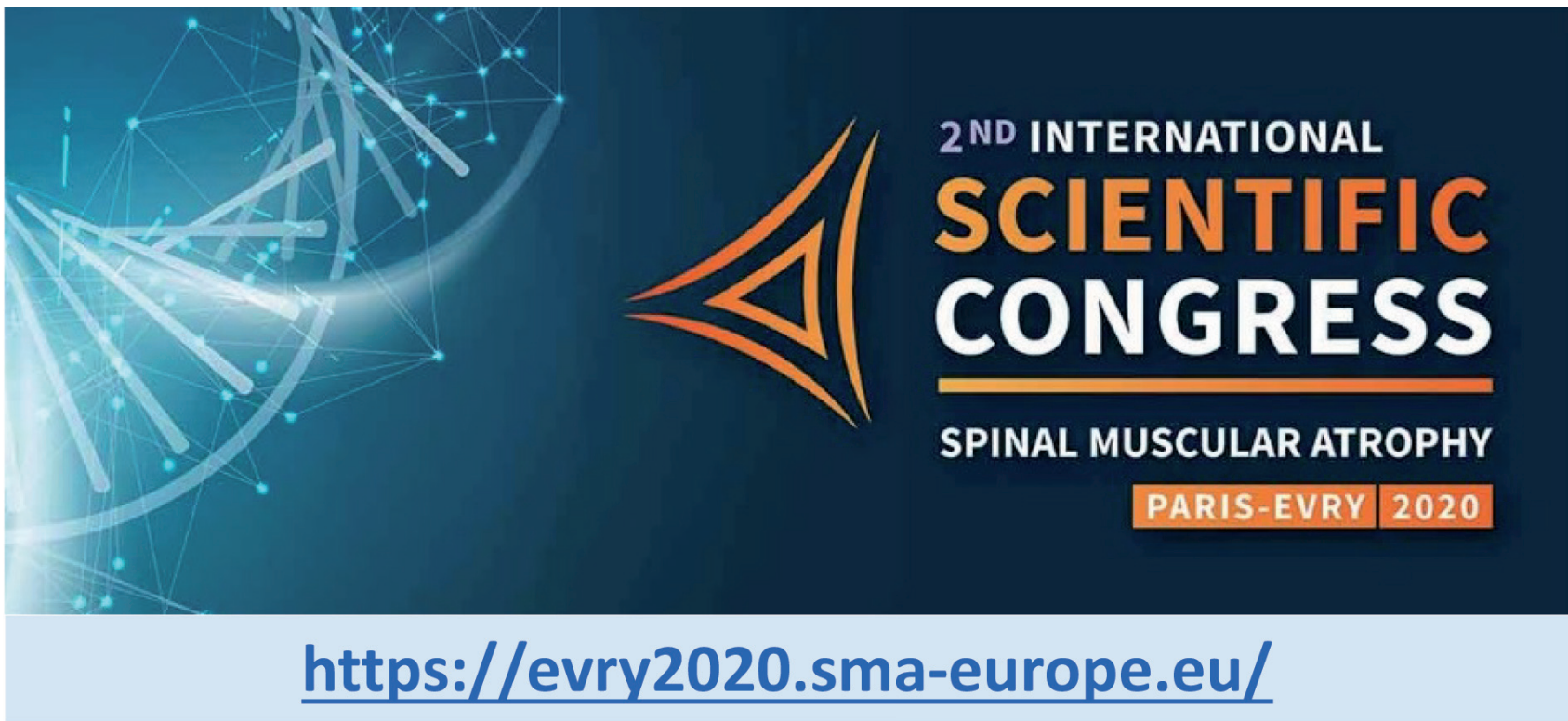

